\title{
Nuclear modification of transverse-longitudinal structure function ratio
}

\author{
M. Ericson* \\ IPN Lyon, IN2P3-CNRS et UCB Lyon I, F-69622 Villeurbanne Cedex, France \\ and Theory Division, CERN, CH-12111 Geneva, Switzerland \\ S. Kumano ${ }^{\dagger}$ \\ Department of Physics, Saga University, Saga 840-8502, Japan
}

(Received 2 December 2002; published 28 February 2003)

\begin{abstract}
We investigate the nuclear effects on the transverse and longitudinal responses in the deep inelastic region due to the nuclear binding and nucleon Fermi motion. We display the role of the transverse-longitudinal admixture due to the transverse nucleon momentum. The mixing effect is appreciable at small $Q^{2}$ values, and gradually disappears at large $Q^{2}$ values. The nuclear modification is then dominated by the binding and Fermi-motion effects which are contained in the spectral function.
\end{abstract}

DOI: 10.1103/PhysRevC.67.022201

PACS number(s): $13.60 . \mathrm{Hb}, 12.38 .-\mathrm{t}, 24.85 .+\mathrm{p}, 25.30 .-\mathrm{c}$

Structure functions in nuclei have been investigated since the discovery of European Muon Collaboration (EMC) effect [1]. Now, as far as the structure functions $F_{2}^{A}$ are concerned, they are relatively well investigated from small to large $x$. The $F_{2}$ structure function contains both longitudinal and transverse components, so that it is interesting to investigate nuclear effects on the separated components.

Experimentally, the nuclear modification of the transverse-longitudinal ratio was first studied by the HERMES Collaboration [2]. The large effect initially suggested was not confirmed by the neutrino data [3] and the HERMES reanalysis [4]. Barone et al. [5] have studied theoretically the longitudinal and transverse shadowing. In addition, the role of the isoscalar meson was investigated by Miller et al. [6].

It is the purpose of this Rapid Communication to discuss, in particular, the influence of the Fermi motion on the two responses and on their ratio. At moderate $x$ values, we show that the modification arises from the admixture of the transverse and longitudinal structure functions. This effect was introduced by Chanfray et al. [7] in the low energy quasielastic regime. The mixing arises from the fact that the photon and nucleon momenta in the laboratory frame are not aligned. We extend here the mixing notion to the deep inelastic regime. There is also an analogous case in spin physics that $g_{1}$ and $g_{2}$ from meson clouds mix [8]. In this paper, we show the admixture of transverse and longitudinal structure functions $F_{1}$ and $F_{L}$. This mechanism produces nuclear modifications of the transverse-longitudinal ratio $R$ at medium and large $x$.

The cross section for electron scattering from a nucleus is given by

$$
\frac{d \sigma}{d E_{e}^{\prime} d \Omega_{e}^{\prime}}=\frac{\left|\vec{p}_{e}^{\prime}\right|}{\left|\vec{p}_{e}\right|} \frac{\alpha^{2}}{\left(q^{2}\right)^{2}} L^{\mu \nu}\left(p_{e}, q\right) W_{\mu \nu}^{A}\left(P_{A}, q\right)
$$

\footnotetext{
*Electronic address: Magda.Ericson@cern.ch

${ }^{\dagger}$ URL: http://hs.phys.saga-u.ac.jp; Electronic address: kumanos @cc.saga-u.ac.jp
}

where $\alpha$ is the fine structure constant, $E_{e}^{\prime}$ and $\Omega_{e}^{\prime}$ are the scattered electron energy and solid angle, and $p_{e}, p_{e}^{\prime}, p_{A}$, and $q$ are initial electron, final electron, nucleus, and virtual photon momenta, respectively, in the nuclear rest frame. In the following discussions, the convention $-g_{00}=g_{11}=g_{22}$ $=g_{33}=+1$ is used so as to have, for example, $p_{A}^{2}=M_{A}^{2}$. The lepton and hadron tensors are denoted as $L^{\mu \nu}$ and $W_{\mu \nu}^{A}$, respectively.

The unpolarized hadron tensor is expressed in terms of two structure functions $W_{1}$ and $W_{2}$ by parity conservation, time-reversal invariance, symmetry under the exchange of the Lorentz indices $\mu$ and $\nu$, and current conservation. The tensors for a nucleus and a nucleon are expressed as

$$
\begin{aligned}
W_{\mu \nu}^{A, N}\left(p_{A, N}, q\right)= & -W_{1}^{A, N}\left(p_{A, N}, q\right)\left(g_{\mu \nu}-\frac{q_{\mu} q_{\nu}}{q^{2}}\right) \\
& +W_{2}^{A, N}\left(p_{A, N}, q\right) \frac{\widetilde{p}_{A, N}^{\mu} \widetilde{p}_{A, N}}{p_{A, N}^{2}}
\end{aligned}
$$

where $\widetilde{p}_{\mu}=p_{\mu}-(p \cdot q) q_{\mu} / q^{2}$. Projection operators of $W_{1}^{A}$ and $W_{2}^{A}$ are, then, defined by

$$
\begin{gathered}
\hat{P}_{1}^{\mu \nu}=-\frac{1}{2}\left(g^{\mu \nu}-\frac{\widetilde{p}_{A}^{\mu} \widetilde{p}_{A}^{\nu}}{\widetilde{p}_{A}^{2}}\right), \\
\hat{P}_{2}^{\mu \nu}=-\frac{p_{A}^{2}}{2 \widetilde{p}_{A}^{2}}\left(g^{\mu \nu}-\frac{3 \widetilde{p}_{A}^{\mu} \widetilde{p}_{A}^{\nu}}{\widetilde{p}_{A}^{2}}\right),
\end{gathered}
$$

so as to satisfy $\hat{P}_{1}^{\mu \nu} W_{\mu \nu}^{A}=W_{1}^{A}$ and $\hat{P}_{2}^{\mu \nu} W_{\mu \nu}^{A}=W_{2}^{A}$.

We describe the nuclear tensor $W_{\mu \nu}^{A}$ by a convolution model with a spectral function for nucleons in a target nucleus:

$$
W_{\mu \nu}^{A}\left(p_{A}, q\right)=\int d^{4} p_{N} S\left(p_{N}\right) W_{\mu \nu}^{N}\left(p_{N}, q\right),
$$

where $p_{N}$ is the nucleon momentum and $S\left(p_{N}\right)$ is the spectral function which indicates the nucleon momentum distri- 
bution in a nucleus. The nuclear structure functions $W_{1}^{A}$ and $W_{2}^{A}$ are projected out by operating $\hat{P}_{1}^{\mu \nu}$ and $\hat{P}_{2}^{\mu \nu}$ on both sides of Eq. (5):

$$
W_{1,2}^{A}\left(p_{A}, q\right)=\int d^{4} p_{N} S\left(p_{N}\right) \hat{P}_{1,2}^{\mu \nu} W_{\mu \nu}^{N}\left(p_{N}, q\right)
$$

Because transverse and longitudinal structure functions are discussed, we specify them in terms of the structure functions $W_{1}$ and $W_{2}$. Photon polarization vectors are given by $\varepsilon_{ \pm}=\mp(0,1, \pm i, 0) / \sqrt{2}$ and $\varepsilon_{0}=\left(\sqrt{\nu^{2}+Q^{2}}, 0,0, \nu\right) / \sqrt{Q^{2}}$, where the photon momentum direction is taken along the $z$ axis $(\vec{q} /|\vec{q}|=\hat{z}), \nu$ is the energy transfer $\nu=E_{e}-E_{e}^{\prime}$, and $Q^{2}$ is defined by $Q^{2}=-q^{2}$. The structure function for the photon polarization $\lambda$ is $W_{\lambda}^{A, N}=\varepsilon_{\lambda}^{\mu *} \varepsilon_{\lambda}^{\nu} W_{\mu \nu}^{A, N}$. Then, the transverse and longitudinal structure functions are given by

$$
W_{T}^{A, N}=\frac{1}{2}\left(W_{+1}^{A, N}+W_{-1}^{A, N}\right), \quad W_{L}^{A, N}=W_{0}^{A, N} .
$$

Taking the nucleus or nucleon rest frame, we have

$$
\begin{gathered}
W_{T}^{A, N}=W_{1}^{A, N}\left(p_{A, N}, q\right), \\
W_{L}^{A, N}=\left(1+\nu_{A, N}^{2} / Q^{2}\right) W_{2}^{A, N}\left(p_{A, N}, q\right)-W_{1}^{A, N}\left(p_{A, N}, q\right),
\end{gathered}
$$

where $\nu_{A} \equiv \nu$, and the photon momentum in the nucleon rest frame is denoted $\left(\nu_{N}, \vec{q}_{N}\right)$ with $\nu_{N}^{2}=\left(p_{N} \cdot q\right)^{2} / p_{N}^{2}$.

Instead of the structure functions $W_{1}$ and $W_{2}, F_{1}$ and $F_{2}$ are usually used in the analysis. They are defined for a nucleus and a nucleon by

$$
\begin{gathered}
F_{1}^{A, N}\left(x_{A, N}, Q^{2}\right)=\sqrt{p_{A, N}^{2}} W_{1}^{A, N}\left(p_{A, N}, q\right), \\
F_{2}^{A, N}\left(x_{A, N}, Q^{2}\right)=\frac{p_{A, N} \cdot q}{\sqrt{p_{A, N}^{2}}} W_{2}^{A, N}\left(p_{A, N}, q\right) .
\end{gathered}
$$

The scaling variables $x_{A}$ and $x_{N}$ are given by

$$
x_{A}=\frac{Q^{2}}{2 p_{A} \cdot q}=\frac{M_{N}}{M_{A}} x, \quad x_{N}=\frac{Q^{2}}{2 p_{N} \cdot q}=\frac{x}{z} .
$$

Here, the Bjorken scaling variable $x$ and the momentum fraction $z$ are defined by

$$
x=\frac{Q^{2}}{2 M_{N} \nu}, \quad z=\frac{p_{N} \cdot q}{M_{N} \nu} .
$$

The transverse structure functions are $F_{1}^{A}$ and $F_{1}^{N}$ for a nucleus and a nucleon, respectively. The longitudinal ones are defined by

$$
\begin{aligned}
F_{L}^{A, N}\left(x_{A, N}, Q^{2}\right)= & \left(1+\frac{Q^{2}}{\nu_{A, N}^{2}}\right) F_{2}^{A, N}\left(x_{A, N}, Q^{2}\right) \\
& -2 x_{A, N} F_{1}^{A, N}\left(x_{A, N}, Q^{2}\right) .
\end{aligned}
$$

The ratio $R_{A}$ of the longitudinal cross section to the transverse one is expressed by the function $R_{A}\left(x_{A}, Q^{2}\right)$ :

$$
R_{A}\left(x_{A}, Q^{2}\right)=\frac{F_{L}^{A}\left(x_{A}, Q^{2}\right)}{2 x_{A} F_{1}^{A}\left(x_{A}, Q^{2}\right)} .
$$

The notations of these structure functions are slightly different from those of the HERMES Collaboration (denoted as H) [2]: $2 x_{A} F_{1}^{A}\left(x_{A}, Q^{2}\right) / A=2 x F_{1}\left(x, Q^{2}\right)_{H}, F_{2}^{A}\left(x_{A}, Q^{2}\right) / A$ $=F_{2}\left(x, Q^{2}\right)_{H}, \quad F_{L}^{A}\left(x_{A}, Q^{2}\right) / A=F_{L}\left(x, Q^{2}\right)_{H}$, and $R_{A}\left(x_{A}\right.$, $\left.Q^{2}\right)=R\left(x, Q^{2}\right)_{H}$. Our structure functions per nucleon are identical to those of the HERMES Collaboration, and the ratio $R_{A}$ is the same.

Calculating Eq. (6) and using the relations in Eq. (10), we obtain expressions for $F_{1}^{A}$ and $F_{2}^{A}$. Writing these expressions in terms of the transverse and longitudinal structure functions, we have

$$
\begin{aligned}
2 x_{A} F_{1}^{A}\left(x_{A}, Q^{2}\right)= & \int d^{4} p_{N} S\left(p_{N}\right) z \frac{M_{N}}{\sqrt{p_{N}^{2}}} \\
& \times\left[\left(1+\frac{\vec{p}_{N \perp}^{2}}{2 \widetilde{p}_{N}^{2}}\right) 2 x_{N} F_{1}^{N}\left(x_{N}, Q^{2}\right)\right. \\
& \left.+\frac{\vec{p}_{N \perp}^{2}}{2 \widetilde{p}_{N}^{2}} F_{L}^{N}\left(x_{N}, Q^{2}\right)\right], \\
F_{L}^{A}\left(x_{A}, Q^{2}\right)= & \int d^{4} p_{N} S\left(p_{N}\right) z \frac{M_{N}}{\sqrt{p_{N}^{2}}} \\
& \times\left[\left(1+\frac{\vec{p}_{N \perp}^{2}}{\widetilde{p}_{N}^{2}}\right) F_{L}^{N}\left(x_{N}, Q^{2}\right)\right. \\
& \left.+\frac{\vec{p}_{N \perp}^{2}}{\widetilde{p}_{N}^{2}} 2 x_{N} F_{1}^{N}\left(x_{N}, Q^{2}\right)\right] .
\end{aligned}
$$

In these expressions, $\vec{p}_{N \perp}$ is the nucleon momentum component perpendicular to the photon one $\vec{q}$, and it is defined by $\vec{p}_{N \perp}=\vec{p}_{N}-\vec{p}_{N \|}$ with $\vec{p}_{N \|}=\left(\vec{p}_{N} \cdot \vec{q}\right) \vec{q} /|\vec{q}|^{2}$. The momentum square $\widetilde{p}_{N}^{2}$ is given by $\widetilde{p}_{N}^{2}=p_{N}^{2}+Q^{2} /\left(4 x_{N}^{2}\right)$.

Equation (15) shows that the longitudinal nucleonic structure function enters into the transverse nuclear one and conversely for the longitudinal nuclear one in Eq. (16). The two mixing coefficients are governed, as expected, by the transverse nucleon momentum, and the admixture terms are proportional to $O\left(\vec{p}_{N \perp}^{2} / Q^{2}\right)$. The mixing is the first cause of modification of the ratio $R_{A}$. Moreover, the usual binding and Fermi-motion effects, which are implicitly contained in our convolution expressions, also introduce a nuclear effect.

The original HERMES effect was reported at small $x$. Their reanalysis indicates that such a large nuclear modification of $R_{A}$ does not exist anymore. Our studies are not directly related to the HERMES finding because the Fermimotion and binding effects become important at medium and large $x$. Our predictions are important for suggesting future experimental efforts for finding such a nuclear modification of $R_{A}$. 
In order to calculate the nuclear structure functions in Eqs. (15) and (16), we need the spectral function for the nucleus. Because ${ }^{14} \mathrm{~N}$ is one of the nuclei investigated by the HERMES Collaboration, we take this nucleus as an example. At this stage, we restrict to the $x$ region, $x<1$, where we may not need a sophisticated model for the ${ }^{14} \mathrm{~N}$ structure. The spectral function is simply described by a shell model:

$$
S\left(p_{N}\right)=\sum_{i}\left|\phi_{i}\left(\vec{p}_{N}\right)\right|^{2} \delta\left(p_{N}^{0}-M_{A}+\sqrt{M_{A-i}^{2}+\vec{p}_{N}^{2}}\right),
$$

where $\phi_{i}$ is the wave function for the $i$ th nucleon, and $M_{A-i}$ is given by $M_{A-i}=M_{A}-M_{N}-\varepsilon_{i}$ with its single particle en$\operatorname{ergy} \varepsilon_{i}$.

The nuclear model should be good enough at least to reproduce gross features of the nucleus, such as nuclear charge radius and binding energy. As such a model, a densitydependent Hartree-Fock model by Bonche, Koonin, and Negele (BKN) [9] is used in the following numerical analysis. In the used BKN code, the Coulomb interaction is turned off, so that the single particle energies and wave functions are the same for the proton and neutron. Using calculated wave functions and energies, we obtain the binding energy and the root-mean-square radius as about $101.7 \mathrm{MeV}$ and $2.54 \mathrm{fm}$. These results agree with the experimental values about $104.66 \mathrm{MeV}$ [10] and $2.45 \mathrm{fm}$ [11], so that the model can be used for our studies of the nuclear structure functions.

Next, we briefly explain the actual integration for evaluating the transverse and longitudinal structure functions in a nucleus. Using the spectral function in Eq. (17) and changing the integration variable to $z$ and $p_{N \perp}$, we have a typical integral of the following form:

$$
\begin{aligned}
& \int d^{4} p_{N} S\left(p_{N}\right) f\left(x / z, Q^{2}\right) \\
& =\sum_{i} \int_{x}^{A} d z \int_{0}^{\infty} d p_{N \perp} \frac{2 \pi p_{N \perp}}{\left|\partial z / \partial p_{N \|}\right|}\left|\phi_{i}\left(p_{N}, \theta_{p}\right)\right|^{2} f\left(x / z, Q^{2}\right),
\end{aligned}
$$

where $p_{N}=\sqrt{p_{N \perp}^{2}+p_{N \|}^{2}}$ and $\theta_{p}=\tan ^{-1}\left(p_{N \perp} / p_{N \|}\right)$. In evaluating the integral, the nucleon energy is taken as $p_{N}^{0}=M_{N}$ $+\varepsilon_{i}-\vec{p}_{N}^{2} /\left(2 M_{A-i}\right)$ by using a nonrelativistic approximation for the recoil nucleus. The momentum fraction $z$ is $z$ $=\left(p_{N}^{0} \nu-p_{N \|} \sqrt{\nu^{2}+Q^{2}}\right) /\left(M_{N} \nu\right)$. Solving these equations, we can express $p_{N}^{0}, p_{N \|}, \partial z / \partial p_{N \|}$ in terms of the integration variables $p_{N \perp}$ and $z$. In this way, the integrations are calculated numerically with the Hartree-Fock wave functions and the following structure functions for a nucleon.

As the leading-order expression of parton distributions, we take those from the MRST (Martin, Roberts, Stirling, and Thorne) parametrization in 1998 [12]. The structure function $F_{2}$ of the proton is calculated by $F_{2}^{p}=\Sigma_{i} e_{i}^{2} x\left(q_{i}+\bar{q}_{i}\right)$. Isospin symmetry is used for the parton distributions in calculating $F_{2}$ of the neutron. Since ${ }^{14} \mathrm{~N}$ is isoscalar and the wave functions are identical for the proton and neutron in the used model, we do not have to take into account the difference

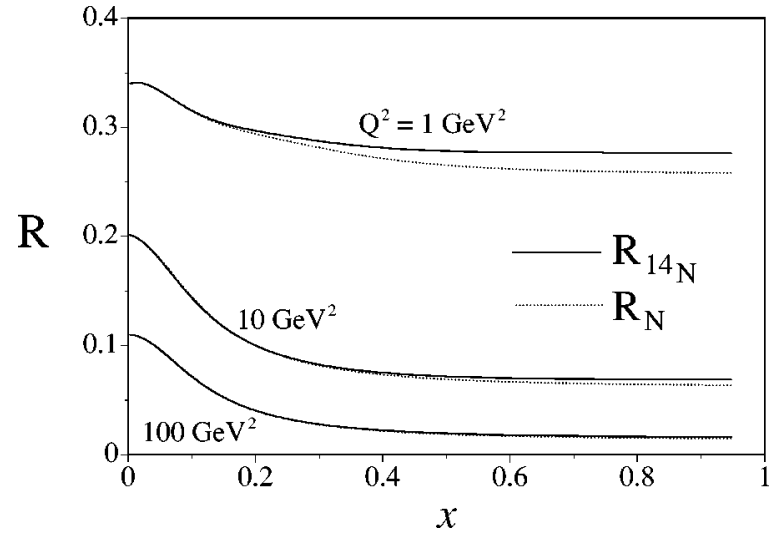

FIG. 1. Transverse-longitudinal structure function ratios $R\left(x, Q^{2}\right)$ at $Q^{2}=1,10$, and $100 \mathrm{GeV}^{2}$. The solid and dotted curves indicate $R_{14_{N}}$ and $R_{N}$, respectively.

between the proton and the neutron structure functions. The nucleon structure function is defined by the average of the proton and neutron structure functions: $F_{2}^{N}=\left(F_{2}^{p}+F_{2}^{n}\right) / 2$. Using this $F_{2}^{N}$ and the SLAC parametrization of 1990 [13] for $R_{N}$, we calculate the transverse and longitudinal structure functions by $F_{1}^{N}=\left(1+Q^{2} / \nu^{2}\right) F_{2}^{N} /\left[2 x\left(1+R_{N}\right)\right]$ and Eq. (13).

The transverse and longitudinal structure functions for the nitrogen nucleus are numerically calculated by Eqs. (15) and (16). Then, the longitudinal-transverse ratio $R_{A}\left(x, Q^{2}\right)$ is evaluated. We should mention that the on-shell structure functions are used for the bound nucleon, whereas off-shell ones should be used in principle [14].

The obtained $R_{1{ }_{1} N}\left(x, Q^{2}\right)$ is displayed in Fig. 1 (solid lines) at $Q^{2}=1,10$, and $100 \mathrm{GeV}^{2}$, together with the nucleon ratio $R_{N}$ (dotted lines). As explained earlier, the admixture corrections are proportional to $O\left(\vec{p}_{N \perp}^{2} / Q^{2}\right)$, so that they are large at small $Q^{2}\left(=1 \mathrm{GeV}^{2}\right)$ in Fig. 1. The corrections increase the ratio $R\left(x, Q^{2}\right)$, and they are conspicuous in the medium and large $x$ regions. They become small at large $Q^{2}\left(=10,100 \mathrm{GeV}^{2}\right)$; however, the overall magnitude of $R\left(x, Q^{2}\right)$ also becomes small. One should note that the $R_{1990}$ is used also outside the applicable range $\left(Q^{2}\right.$ $<30 \mathrm{GeV}^{2}$ ).

In Fig. 2, we show the nitrogen-nucleon ratio $R_{14}\left(x, Q^{2}\right) / R_{N}\left(x, Q^{2}\right)$ at $Q^{2}=1,10$, and $100 \mathrm{GeV}^{2}$. The solid curves are the ratios with the full nuclear modification. The dashed ones are obtained by suppressing the $\vec{p}_{N \perp}^{2}$ terms in Eqs. (15) and (16), i.e., in particular we have suppressed the admixture effect. Without the admixture, the nuclear modification remains small at $Q^{2}=1 \mathrm{GeV}^{2}$. However, because of the nucleon transverse motion, the transverselongitudinal admixture gives rise to about $5 \%$ positive modification at medium $x(\approx 0.5)$. This $5 \%$ effect is consistent with the value of the mixing coefficient $\vec{p}_{N \perp}^{2} / \widetilde{p}_{N}^{2}$ $\approx 4 x_{N}^{2}\left\langle\vec{p}_{N \perp}^{2}\right\rangle / Q^{2} \approx 0.04$. At larger $Q^{2}\left(=10\right.$ and $\left.100 \mathrm{GeV}^{2}\right)$, the admixture effects become smaller. The factor $\vec{p}_{N \perp}^{2} / Q^{2}$ becomes small; however, the ratio $2 x_{N} F_{1} / F_{L}^{N}$ becomes large 


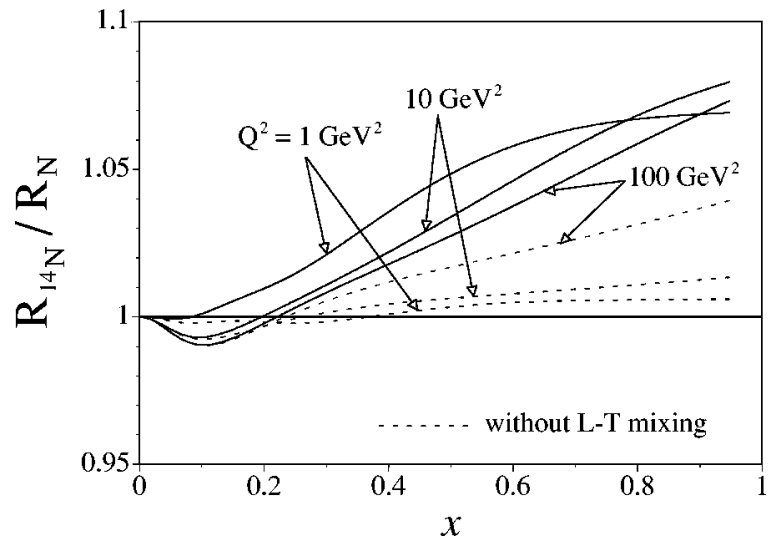

FIG. 2. The solid curves show the transverse-longitudinal ratios $R_{A}\left(x, Q^{2}\right)$ for ${ }^{14} \mathrm{~N}$ divided by the corresponding ratio $R_{N}\left(x, Q^{2}\right)$ for the nucleon at $Q^{2}=1,10$, and $100 \mathrm{GeV}^{2}$. The dashed curves are obtained without the longitudinal-transverse admixture.

$2 x_{N} F_{1} / F_{L}^{N}=14$ and 53 for $Q^{2}=10$ and $100 \mathrm{GeV}^{2}$, respectively, at $x_{N}=0.5$. Therefore, the overall admixture correction is of the order of 3 and $1 \%$ for $Q^{2}=10$ and $100 \mathrm{GeV}^{2}$, respectively. The dashed curves in Fig. 2 indicate that the correction from the spectral function also contributes to the modification.

Using a convolution description of nuclear structure functions, we have derived the nuclear modification of the longitudinal-transverse structure function ratio $R\left(x, Q^{2}\right)$ at medium and large $x$. We have found that the conventional convolution description of the nuclear structure functions leads to the nuclear modification of the transverselongitudinal structure function ratio $R\left(x, Q^{2}\right)$. The physical origin of the modification has been clarified in this Rapid Communication. The most important point is the transverselongitudinal admixture of the nuclear structure functions due to the nucleon momentum transverse to the virtual photon momentum. The mixing effects we found are moderate at small $Q^{2}$ and disappear at large $Q^{2}$. The nuclear effects are then dominated by the binding and Fermi-motion effects contained implicitly in the convolution expression.

In this work, we have used the simplest convolution model. In particular, we have not included the effects of short-range nucleon correlation and off-shell structure functions, and they are expected to be important at large $x$. A better description of the nuclear dynamics is necessary to extend this work to the large $x$ domain.
[1] For a summary, see D. F. Geesaman, K. Saito, and A. W. Thomas, Annu. Rev. Nucl. Part. Sci. 45, 337 (1995).

[2] K. Ackerstaff, HERMES Collaboration, et al., Phys. Lett. B 475, 386 (2000); A. Airapetian et al., hep-ex/0210067, erratum.

[3] U. K. Yang, CCFR/NuTeV Collaboration, et al., Phys. Rev. Lett. 87, 251802 (2001).

[4] A. Airapetian, HERMES Collaboration, et al., hep-ex/0210068.

[5] V. Barone and M. Genovese, hep-ph/9610206; B. Kopeliovich, J. Raufeisen, and A. Tarasov, Phys. Rev. C 62, 035204 (2000).

[6] G. A. Miller, S. J. Brodsky, and M. Karliner, Phys. Lett. B 481, 245 (2000); G. A. Miller, Phys. Rev. C 64, 022201 (2001).

[7] G. Chanfray, J. Delorme, M. Ericson, and A. Molinari, Nucl. Phys. A556, 439 (1993).
[8] S. Kumano and M. Miyama, Phys. Rev. D 65, 034012 (2002).

[9] P. Bonche, S. Koonin, and J. W. Negele, Phys. Rev. C 13, 1226 (1976); a computer code was supplied by A. S. Umar.

[10] G. Audi and A. H. Wapstra, Nucl. Phys. A595, 409 (1995).

[11] M. A. Preston and R. K. Bhaduri, Structure of the Nucleus (Addison-Wesley, New York, 1975), p. 99.

[12] A. D. Martin, R. G. Roberts, W. J. Stirling, and R. S. Thorne, Eur. Phys. J. C 4, 463 (1998); the computer code is taken from the website, http://www-spires.dur.ac.uk/hepdata/pdf.html

[13] L. W. Whitlow, S. Rock, A. Bodek, S. Dasu, and E. M. Riordan, Phys. Lett. B 250, 193 (1990); the SLAC parametrization $R_{1990}$ is defined by the average over three functions in L. W. Whitlow, report SLAC-357, 1990, p. 109.

[14] S. A. Kulagin, G. Piller, and W. Weise, Phys. Rev. C 50, 1154 (1994); S. Liuti and F. Gross, Phys. Lett. B 356, 157 (1995). 